\title{
DISTRIBUTION DU COUT D'UN SINISTRE DANS L'ASSURANCE SOCIALE CONTRE LES ACCIDENTS
}

\author{
R. LATSCHA \\ Lucerne
}

I. On n'a guère entendu dire jusqu'ici que la distribution empirique du coût d'un sinistre dans l'assurance sociale en cas d'accidents ait fait l'objet d'études spéciales. Comme la Caisse nationale suisse d'assurance en cas d'accidents est en train de faire de tels examens en vue de résoudre certains problèmes de tarification que pose l'application de la théorie du risque, nous avons pensé qu'il serait intéressant de présenter une courte communication à ce sujet ${ }^{1}$ ). Le présent rapport a pour objet l'étude des fonctions empiriques de la distribution du coût d'un accident professionnel dans cinq branches industrielles et artisanales en Suisse ${ }^{2}$ ). Nous voudrions faire précéder notre exposé de quelques considérations sur l'organisation et les tâches de l'assurance sociale contre les accidents en Suisse ainsi que de l'énumération des désignations et abréviations employées dans ce rapport.

2. La Caisse nationale suisse d'assurance en cas d'accidents à Lucerne, en fonction depuis I9I8, est un établissement de droit public autonome. Elle assure obligatoirement contre les conséquences économiques des accidents professionnels et non professionnels les ouvriers et employés des entreprises soumises à l'assurance conformément à la loi sur l'assurance en cas de maladie et d'accidents de rgII. Sont considérés comme accidents professionnels les accidents qui se produisent pendant le travail dans l'entreprise

\footnotetext{
1) En complément de ce rapport, nous renvoyons encore alux publications suivantes qui traitent de questions en rapport avec la distribution du coût d'un accident:

R. Latscha: Zur Anwendung der kollektiven Risikotheorie in der schweizerischen obligatorischen Unfallversicherung. Bulletin de l'Association des actuaires suisses, vol. 56,1956 .

Caisse nationale suisse d'assurance en cas d'accidents: Résultats de la statistique des accidents de la huitième période quinquennale 1953-1957.

2) Les examens statistiques et leur présentation ont été faits par les soins de notre collaborateur, M. H. Koch; nous tenons à l'en remercier ici.
} 
ainsi que les maladies professionnelles. Tous les autres accidents, notamment ceux qui se produisent sur le chemin pour se rendre au travail et en revenir, sont des accidents non professionnels. L'assurance des accidents professionnels et celle des accidents non professionnels forment deux branches d'assurance distinctes. Dans les deux branches, les prestations d'assurance sont les mêmes. Elles consistent dans le paiement des frais de traitement, d'une indemnité de chômage ainsi que dans l'octroi de rentes d'invalidité et de survivants.

Frais de traitement: Le sinistré reçoit les soins médicaux, les médicaments et les autres moyens nécessaires à la guérison. Les frais en sont payés en plein et sans limitation par l'assurance, l'assuré ayant le libre choix du médecin.

Indemnité de chomage: A partir du troisième jour dès l'accident, le sinistré touche le $80 \%$ de son salaire à titre d'indemnité de chômage; le salaire assuré, soit le salaire soumis au paiement des primes et déterminant pour le montant de l'indemnité de chômage, comporte un maximum fixé par la loi. Le salaire maximum assuré est adapté de temps à autre au niveau des salaires. Il s'est élevé successivement, par jour et par assuré, à 26 francs suisses de I945-I952, à 30 fr.s. de I953-I956 et à 40 fr.s. depuis 1957 .

Cô̂t des rentes: Les rentes d'invalidité sont graduées suivant le degré de l'invalidité et, pour une invalidité de I00\%, s'élèvent à $70 \%$ du salaire assuré. Les rentes de survivants se montent au total à $60 \%$ du maximum du salaire assuré et sont versées aux veuves, orphelins, aux parents en ligne ascendante et aux frères et soeurs encore mineurs.

Les prestations de l'assurance-accidents obligatoire ne sont pratiquement pas limitées - à cause de la prise en charge totale des frais médicaux - et cela est d'autant plus vrai que les nombreuses rechutes dues à des accidents donnent droit ultérieurement à des prestations d'assurance. Cette couverture très large du risque provoque des variations d'une amplitude considérable de la chargeaccidents qui, comme l'expérience l'a montré, peut atteindre plus de 300.000 fr.s. par accident. Pratiquement tout accident occasionne des frais de traitement; des indemnités de chômage sont allouées 
dans la moitié environ et des rentes dans le $2 \%$ seulement des accidents. En gros, les frais de traitement représentent $r / 5$, les indemnités de chômage $\mathrm{I} / 3$ et le coût des rentes la moitié de la charge totale.

3. Désignations et abréviations:

Les composantes de la charge sont les frais de traitement (T), l'indemnité de chômage $(C, c)$, le coût des rentes $(R, r)$ ainsi que le coût total.

Les catégories d'accidents sont désignées par des grandes et petites lettres suivant que la composante correspondante de la charge est représentée ou pas. Pour simplifier nous admettons que tout accident comprend des frais de traitement.

\begin{tabular}{|cl|}
\hline Catégories simples & \multicolumn{1}{c|}{ Catégories composées } \\
$\mathrm{TCr}$ & $\mathrm{TC}=\mathrm{TCr}+\mathrm{TCR}$ \\
$\mathrm{Tcr}$ & $\mathrm{Tc}=\mathrm{Tcr}+\mathrm{TcR}$ \\
$\mathrm{TCR}$ & $\mathrm{TR}=\mathrm{TCR}+\mathrm{TcR}$ \\
$\mathrm{TcR}$ & $\mathrm{Tr}=\mathrm{TCr}+\mathrm{Tcr}$ \\
& $\mathrm{T}=\mathrm{TCr}+\mathrm{Tcr}+\mathrm{TCR}+\mathrm{TcR}$ \\
\hline
\end{tabular}

Exemples:

TCr: accidents avec frais de traitement et indemnité de chômage, mais sans rente

TABLE

Nombre et coût des

\begin{tabular}{|c|c|c|c|c|c|c|c|}
\hline \multirow{2}{*}{ Groupes } & \multirow{2}{*}{ Années } & \multirow{2}{*}{$\begin{array}{c}\text { Nombre } \\
\text { moyen } \\
\text { annuel } \\
\text { des assurés }\end{array}$} & \multicolumn{5}{|c|}{ Nombre des accidents } \\
\hline & & & $\mathrm{TCr}$ & $\mathbf{T} c \mathbf{r}$ & TCR & TcR & $\mathrm{T}$ \\
\hline I Construction de machines & I 949-I 95 I & 42000 & I 3194 & I6 838 & 432 & 27 & $3049 \mathrm{I}$ \\
\hline 2 Fonderies $\ldots \ldots \ldots \ldots \ldots$ & I949-I952 & 12500 & 7920 & 7 II 5 & 258 & Io & I 5303 \\
\hline 3 Bâtiment $\ldots \ldots \ldots \ldots \ldots \ldots \ldots$ & $1949-1950$ & 52000 & 27770 & I7 039 & 816 & 59 & 45684 \\
\hline galeries $\quad \ldots \ldots \ldots \ldots \ldots$ & I95I-1953 & $6 \mathrm{I} 100$ & 9466 & 4607 & 443 & 83 & I4 599 \\
\hline 5 Carrières $\ldots \ldots \ldots \ldots \ldots \ldots \ldots$ & I949-I952 & 5600 & 4600 & 2250 & 209 & 37 & 7096 \\
\hline
\end{tabular}

3) Compte tenu des prestations ultérieures versées jusqu'en r 956 . 
Tcr: accidents avec frais de traitement seulement

TC: accidents avec frais de traitement et indemnité de chômage

Tc: accidents avec frais de traitement, sans indemnité de chômage

T: accidents avec frais de traitement, c'est-à-dire l'ensemble des accidents.

Fonctions de distribution:

$u(z)$ : Fonction des fréquences des frais normalisés $z$ d'un accident. $u(z)$ correspond à la probabilité que les frais normalisés d'un accident seront exactement de $z$.

$U(z)=\sum_{\zeta=0}^{z} u(\zeta):$

Fonction de distribution des frais normalisés d'un accident. $U(z)$ correspond à la probabilité que les frais normalisés d'un accident seront inférieurs à $z$.

$S(z)=\frac{\sum_{\zeta=0}^{z} \zeta u(\zeta)}{\sum_{\zeta=0}^{\infty} \zeta u(\zeta)} \mathrm{IOO}:$

„Fonction de distribution" des frais normalisés de tous les accidents exprimant que le $S(z) \%$ de la somme totale des frais est dû aux accidents dont les frais sont inférieurs à $z$.

4. Nous montrons maintenant quelles sont, pour des intervalles de Io francs, les fonctions empiriques de distribution des frais d'un accident professionnel dans 5 branches industrielles et artisanales

I

accidents professionnels

\begin{tabular}{|c|c|c|c|c|c|c|c|c|c|c|c|c|c|c|c|}
\hline \multicolumn{16}{|c|}{ Coût des accidents ${ }^{3}$ ) (en Iooo fr.s.) } \\
\hline \multicolumn{5}{|c|}{ Frais de traitement } & \multicolumn{3}{|c|}{$\begin{array}{l}\text { Indemnité de } \\
\text { chômage }\end{array}$} & \multicolumn{3}{|c|}{$\begin{array}{l}\text { Coût des } \\
\text { rentes }\end{array}$} & \multicolumn{5}{|c|}{ Coût total } \\
\hline $\mathrm{TCr}$ & Ter & TCR & TcR & $\mathrm{T}$ & $\mathrm{TCr}$ & TCR & $\mathrm{T}$ & TCR & $\mathrm{TcR}$ & $\mathbf{T}$ & $\mathrm{TCr}$ & Tcr & TCR & $\mathrm{TcR}$ & $\mathrm{T}$ \\
\hline I $07 \mathrm{I}$ & 356 & $24 \mathrm{I}$ & 3 & 1671 & 2574 & $52 \mathrm{I}$ & 3095 & 2646 & I 225 & $387 \mathrm{r}$ & 3645 & 356 & 3408 & I 228 & 8637 \\
\hline 697 & 163 & 227 & I & I 088 & I 771 & 388 & $2 I 59$ & 2396 & 443 & 2839 & 2468 & I63 & 3 O1I & 444 & \\
\hline 2410 & 385 & I 019 & 9 & 3823 & 6553 & 1920 & 8473 & $825 \mathrm{I}$ & I 587 & 9838 & 8963 & 385 & II I 90 & 1596 & 22134 \\
\hline I $3^{89}$ & I I I & 838 & I9 & 2357 & 3034 & I 315 & 4349 & 6337 & 3524 & $986 \mathrm{r}$ & 4423 & I I I & 8490 & 3543 & 16567 \\
\hline 524 & 53 & 254 & 7 & 838 & I 182 & 366 & I 548 & 2 II 4 & 980 & 3094 & 1706 & 53 & 2734 & 987 & 5480 \\
\hline
\end{tabular}


TABLE

Fonctions de distribution $\mathrm{U}(\mathrm{z})$ (en pour

\begin{tabular}{|c|c|c|c|c|c|c|c|c|c|c|c|c|c|c|c|}
\hline \multirow{2}{*}{$\begin{array}{c}\text { Frais } \\
\text { normalisés } \\
z\end{array}$} & \multicolumn{9}{|c|}{ Frais de traitement } & \multicolumn{6}{|c|}{ Indemnité de chômage } \\
\hline & $\mathrm{T}$ & $\mathrm{TCr}$ & Ter & TCR & TcR & $\mathrm{TC}$ & $\mathrm{Tc}$ & TR & $\mathrm{Tr}$ & $\mathrm{T}$ & $\mathrm{TCr}$ & TCR & $\mathrm{TC}$ & $\mathrm{TR}$ & $\operatorname{Tr}$ \\
\hline 0 & $\circ$ & 0 & 0 & 0 & II & 0 & o & 0 & o & 55 & o & 0 & 0 & 6 & 56 \\
\hline 0,05 & 2 & o & 2 & o & II & 0 & 2 & I & 2 & 56 & 4 & 2 & 5 & 8 & 57 \\
\hline $0, \mathbf{I}$ & 5 & 0 & 3 & I & I 2 & 0 & 3 & 3 & 4 & 57 & 9 & 5 & Io & II & 58 \\
\hline 0,15 & 7 & 0 & 5 & 3 & 13 & I & 5 & 6 & 6 & 58 & 14 & 7 & I6 & 13 & 59 \\
\hline 0,2 & 12 & I & 7 & 7 & I 4 & 2 & 7 & IO & 9 & 59 & 18 & 9 & 20 & 14 & 60 \\
\hline 0,4 & 39 & 17 & 13 & 42 & $2 \mathrm{I}$ & 27 & 13 & 42 & 32 & 63 & 32 & 22 & 36 & 25 & 63 \\
\hline 0,6 & 57 & 44 & 28 & 58 & 36 & 54 & 28 & 59 & 50 & 66 & 46 & 43 & $5 \mathrm{I}$ & 43 & 66 \\
\hline 0,8 & 68 & 64 & 47 & 66 & 55 & $7 \mathrm{I}$ & 47 & 66 & 63 & 69 & 58 & 58 & 63 & 57 & 69 \\
\hline I & 76 & 75 & 64 & 73 & $7 I$ & 79 & 64 & 73 & 72 & 73 & 68 & 68 & $7 \mathrm{I}$ & 68 & $7 \mathrm{II}$ \\
\hline 1,2 & 82 & 82 & 74 & 77 & 77 & 84 & 74 & 78 & 78 & 76 & 74 & 76 & 77 & 74 & 74 \\
\hline $\mathrm{r}, 4$ & 86 & 86 & 84 & 79 & $8 \mathrm{I}$ & 87 & 84 & 80 & 83 & 79 & 80 & 81 & 82 & 80 & 77 \\
\hline $\mathrm{I}, 6$ & 89 & 89 & 89 & 82 & 82 & 89 & 89 & 83 & 87 & $8 I$ & 83 & 85 & 85 & 84 & 79 \\
\hline $\mathrm{I}, 8$ & 90 & $9 I$ & 92 & 85 & 84 & $9 \mathrm{I}$ & 92 & 85 & 89 & 83 & 86 & 88 & 88 & 87 & 82 \\
\hline 2 & 92 & 92 & 94 & 87 & 85 & 92 & 94 & 87 & gI & 85 & 89 & 90 & 90 & 90 & 83 \\
\hline 4 & 97 & 97 & 98 & 97 & 100 & 97 & 98 & 97 & 97 & 94 & 97 & 97 & 97 & 97 & 94 \\
\hline 6 & 98 & 98 & 99 & 98 & & 98 & 99 & 98 & 98 & 97 & 99 & 99 & 98 & 99 & 97 \\
\hline 8 & 99 & 99 & 99 & 99 & & 99 & 99 & 99 & 99 & 98 & 99 & 99 & 99 & 99 & 98 \\
\hline IO & 99 & 99 & 99 & 99 & & 99 & 99 & 99 & 99 & 99 & 99 & 100 & 99 & IOO & 99 \\
\hline 20 & 99 & 99 & 99 & IOO & & 99 & 99 & 100 & 99 & 99 & 99 & & 99 & & 99 \\
\hline 40 & 99 & 99 & 99 & & & 99 & 99 & & 99 & 99 & 99 & & 99 & & 99 \\
\hline 60 & 99 & 99 & IOO & & & 99 & 100 & & 99 & 99 & 99 & & 100 & & 99 \\
\hline 80 & 99 & 99 & & & & 99 & & & 99 & 99 & 100 & & & & 99 \\
\hline IOO & 99 & 99 & & & & 100 & & & 99 & 99 & & & & & 99 \\
\hline 150 & 99 & 100 & & & & & & & 99 & 100 & & & & & 99 \\
\hline 200 & 100 & & & & & & & & 100 & & & & & & I0o \\
\hline 300 & & & & & & & & & & & & & & & \\
\hline 700 & & & & & & & & & & & & & & & \\
\hline 900 & & & & & & & & & & & & & & & \\
\hline 1000 & & & & & & & & & & & & & & & \\
\hline Moyennes & 55 & $8 \mathrm{I}$ & $2 \mathrm{I}$ & 556 & 97 & 96 & $2 I$ & 529 & 47 & IOI & 195 & 1207 & 228 & $I 136$ & 86 \\
\hline & & & & & & & & & & & & & & & \\
\hline Ampli- & & & & & & & & & & & & & & & \\
\hline tudes de & IO 000 & 10000 & I 300 & 7500 & 350 & 10 ooo & I 300 & 7500 & I0 000 & I5 000 & 15000 & 12000 & 15000 & 12000 & 15000 \\
\hline $\begin{array}{l}\text { variation } \\
\text { (en fr.s.) }\end{array}$ & & & & & & & & & & & & & & & \\
\hline
\end{tabular}

spécialement choisies. Nous soulignons qu'il s'agit uniquement des accidents professionnels dont le coût - à la différence des accidents non professionnels - dépend dans une large mesure du niveau du risque et des salaires dans la branche industrielle ou artisanale considérée. Le matériel statistique de base a été préparé à l'aide des cartes perforées. Le coût des prestations payées ultérieurement 
cent) pour la construction de machines

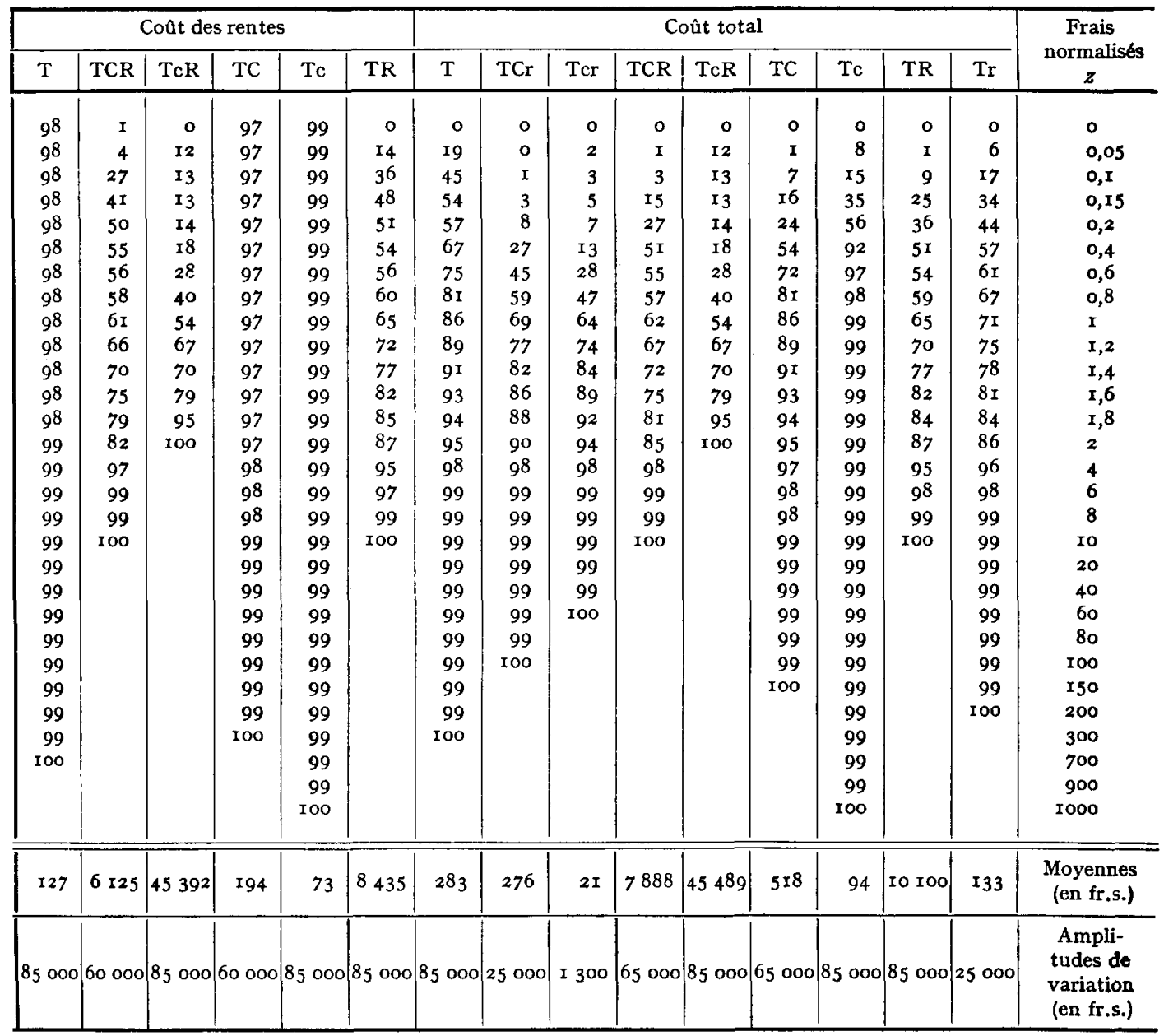

a été englobé avec celui du cas initial. Ces paiements sont naturellement très nombreux dans les années qui suivent immédiatement l'accident.

La table I (p. 64-65) donne un aperçu général du nombre et du coût des accidents professionnels. Le coût des accidents ne comprend pas les frais causés par la silicose en tant que maladie professionnelle. 
Le coût des rentes correspond aux réserves mathématiques des rentes allouées (le sytème des réserves mathématiques est prescrit par la loi) ainsi que les indemnités en capital versées pour le rachat de petites rentes d'invalidité transitoires.

La table 2 (p. 66-67) contient les fonctions de distribution $U(z)$ des frais normalisés d'un accident dans l'industrie des machines. La note en bas donne, outre les amplitudes de variation non normalisées, les moyennes de normalisation, calculées sur la base de la table I; ainsi pour l'indemnité de chômage (catégorie TC): $228=(2574000+52$ I 000 $):($ I 3 I $94+432)$. La table 2 nous indique par exemple que dans le $5 \mathrm{I} \%$ de tous les accidents avec indemnité de chômage, cette indemnité est plus petite que 0,6 fois la moyenne de l'indemnité de chômage de ces accidents, c'est-à-dire plus petite que $0,6.228 \sim$ I37 fr.s.

Les frais de traitement se répartissent plus au moins dans la même proportion dans toutes les catégories (exception $T c R$ : II \% ou 3 de ces cas en soi peu fréquents n'ont pas donné lieu à des frais de traitement. Il s'agit de rentes de survivants dans des cas où la mort est survenue immédiatement). Pour les autres composantes, les valeurs présentent une certaine concordance, ce qu'il faut attribuer soit au nombre relativement petit des cas de rentes, soit au fait que ces cas donnent presque toujours lieu au paiement d'une indemnité de chômage. On constate des fonctions à peu près de même allure, pour l'indemnité de chômage entre les catégories $T$ et $T r, T C r$ et $T C$ ainsi qu'entre $T C R$ et $T R$, pour les ventes entre $T, T C$ et $T c$ d'une part et $T C R$ et $T R$ d'autre part. Pour le coût total aussi, on constate certaines constantes. Si l'on construit la courbe du coût total en utilisant une échelle logarithmique pour $z$, on obtient pour $T c$ et $T c r$ ainsi que pour $T C$ et $T C r$ des courbes d'allure semblable avec toutefois un déplacement de leurs phases.

La table 3 (p. 69) contient les fonctions de distribution $U(z)$ des frais totaux normalisés d'un accident et celles $S(z)$ de la somme totale des frais normalisés des accidents. Sa lecture nous montre que, pour la construction des machines par exemple, dans le $9 \mathrm{I} \%$ des cas le coût total d'un accident ne dépasse pas I,4 fois la moyenne, soit I,4. $283 \sim 400$ fr.s. De plus ce $9 \mathrm{I} \%$ des accidents ne cause en 
tout que le $25 \%$ de la somme des frais de tous les accidents.

Quoique les distributions $U(z)$ et $S(z)$ ne présentent en général pas de grandes différences d'un groupe à l'autre, on peut noter une concordance particulièrement marquée entre les groupes I et 2 respectivement 4 et 5 . Cela provient probablement du fait qu'il

TABLE 3

Fonctions de distribution $\mathrm{U}(\mathrm{z})$ et $\mathrm{S}(\mathrm{z})$ (en pour-cent)

\begin{tabular}{|c|c|c|c|c|c|c|c|c|c|c|c|c|}
\hline \multirow{2}{*}{$\begin{array}{c}\text { Frais } \\
\text { normalisés } \\
z\end{array}$} & \multicolumn{2}{|c|}{$\begin{array}{c}\quad 1 \\
\text { Construction } \\
\text { de machines }\end{array}$} & \multicolumn{2}{|c|}{$\begin{array}{c}2 \\
\text { Fonderies }\end{array}$} & \multicolumn{2}{|c|}{$\begin{array}{c}3 \\
\text { Bâtiment }\end{array}$} & \multicolumn{2}{|c|}{$\begin{array}{c}\mathbf{4} \\
\text { Construction } \\
\text { de tunnels et } \\
\text { de galeries }\end{array}$} & \multicolumn{2}{|c|}{$\begin{array}{c}5 \\
\text { Carrières }\end{array}$} & \multicolumn{2}{|c|}{$\left.I-5^{*}\right)$} \\
\hline & $U(z)$ & $S(z)$ & $U(z)$ & $S(z)$ & $(U z)$ & $S(z)$ & $U(z)$ & $S(z)$ & $U(z)$ & $S(z)$ & $U(z)$ & $S(z)$ \\
\hline 0 & 0 & 0 & $\circ$ & 0 & 0 & o & 0 & 0 & 0 & 0 & 0 & 0 \\
\hline 0,05 & 19 & I & 25 & I & 26 & o & $3 I$ & 0 & 30 & o & 22 & I \\
\hline $0, I$ & 45 & 2 & 43 & 2 & 36 & $\mathbf{I}$ & 37 & I & 35 & I & 37 & 2 \\
\hline 0,15 & 54 & 3 & 47 & 3 & 40 & 2 & 46 & 2 & $4 I$ & 2 & 47 & 4 \\
\hline 0,2 & 57 & 4 & $5 \mathrm{I}$ & 3 & 44 & 3 & 56 & 4 & 50 & 4 & 54 & 5 \\
\hline 0,4 & 67 & 7 & 64 & 7 & 64 & 9 & 79 & IO & 77 & II & 72 & Io \\
\hline 0,6 & 75 & I I & 75 & 13 & 78 & 15 & 86 & 14 & 86 & I 5 & $8 \mathrm{I}$ & I3 \\
\hline 0,8 & 81 & I5 & 82 & I 8 & 85 & 20 & 90 & I7 & 90 & 18 & 86 & I5 \\
\hline I & 86 & I9 & 87 & 22 & 89 & 24 & 92 & I 8 & 92 & 20 & 89 & I7 \\
\hline 1,2 & 89 & 23 & 90 & 25 & $9 \mathrm{I}$ & 27 & 93 & 20 & 93 & $2 I$ & $9 \mathrm{I}$ & 19 \\
\hline $\mathrm{I}, 4$ & $9 I$ & 25 & 92 & 28 & 93 & 29 & 94 & $2 \mathrm{I}$ & 94 & 22 & 93 & 20 \\
\hline 1,6 & 93 & 28 & 94 & 30 & 94 & 30 & 95 & 22 & 95 & 23 & 94 & 21 \\
\hline I, 8 & 94 & 30 & 94 & 32 & 95 & 32 & 95 & 22 & 95 & 24 & 95 & 22 \\
\hline 2 & 95 & $3 I$ & 95 & 33 & 96 & 33 & 96 & 23 & 95 & 25 & 96 & 23 \\
\hline 4 & 98 & 40 & $9^{8}$ & $4 \mathrm{I}$ & 98 & 39 & 97 & 27 & 97 & 28 & 97 & $3^{\circ}$ \\
\hline 6 & 99 & 44 & 99 & 44 & 98 & $4 I$ & 97 & 28 & 97 & 30 & 98 & 34 \\
\hline 8 & 99 & 47 & 99 & 45 & $9^{8}$ & 43 & $9^{8}$ & 30 & 97 & 33 & 99 & 37 \\
\hline IO & 99 & 48 & 99 & 46 & 99 & 44 & $9^{8}$ & $3 I$ & 98 & 34 & 99 & 39 \\
\hline 20 & 99 & $5 I$ & 99 & 48 & 99 & 49 & 99 & 45 & 99 & 50 & 99 & 49 \\
\hline 40 & 99 & 59 & 99 & 61 & 99 & 66 & 99 & 70 & 99 & 75 & 99 & 63 \\
\hline 60 & 99 & 69 & 99 & 73 & 99 & 80 & 99 & 80 & 99 & 85 & 99 & 72 \\
\hline 80 & 99 & 77 & 99 & $8 I$ & 99 & 88 & 99 & 94 & 99 & 96 & 99 & 79 \\
\hline 100 & 99 & $8 I$ & 99 & 84 & 99 & 93 & 99 & 99 & 99 & 98 & 99 & 85 \\
\hline 150 & 99 & 85 & 99 & 92 & IOO & 100 & 100 & 100 & IOO & IOO & 99 & 95 \\
\hline 200 & 99 & $9 \mathrm{I}$ & 99 & 99 & & & & & & & IOO & 100 \\
\hline 250 & 99 & 95 & 100 & 100 & & & & & & & & \\
\hline 300 & 100 & 100 & & & & & & & & & & \\
\hline $\begin{array}{l}\text { Moyennes } \\
\text { (en fr.s.) }\end{array}$ & - & 283 & . & 398 & . & 484 & . & I I 35 & . & 772 & . & 520 \\
\hline $\begin{array}{l}\text { Amplitudes } \\
\text { de variation } \\
\text { (en fr.s.) }\end{array}$ & . & 85000 & . & 90000 & . & 79000 & . & 147000 & . & 87000 & . & . \\
\hline
\end{tabular}

*) Ajustement graphique (voir graphique page 9) 
s'agit de groupes qui présentent des analogies au point de vue technique et dans lesquels la structure du processus des accidents est semblable. Dans le graphique suivant, auquel correspondent les fonctions figurant dans la dernière colonne double de la table 3 , on a réuni et ajusté les courbes des cinq groupes. Pour les besoins du graphique, nous avons choisi pour $z$ l'échelle logarithmique. En moyenne p. ex. $76 \%$ de tous les accidents présentent des frais normalisés toujours inférieurs à $z=0,5$. La somme des frais de ce $76 \%$ des accidents ne représente au total que II $\%$ de la somme des frais de tous les accidents. Un fait à relever est la symétrie centrale de la courbe $U(z)$.

La table 4 (p. 70-7I) donne les paramètres des fonctions empiriques $u(z)$ pour tous les groupes, composantes et catégories:

$$
\text { Moyenne }=m_{1}=\sum_{z=0}^{\infty} z u(z)
$$

TABLE

Parametres des fonctions

\begin{tabular}{|c|c|c|c|c|c|c|c|c|c|c|c|c|c|c|c|}
\hline \multirow{2}{*}{ Groupes } & \multicolumn{9}{|c|}{ Frais de traitement } & \multicolumn{6}{|c|}{ Indemnité de chômage } \\
\hline & $T$ & $T C r$ & $T c r$ & $T C R$ & $T c R$ & $T C$ & $T c$ & $T R$ & $T r$ & $T$ & $T C r$ & $T C R$ & $T C$ & $T R$ & $T r$ \\
\hline \multirow{6}{*}{ 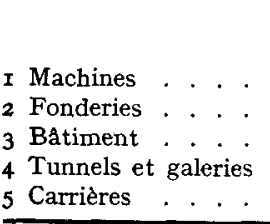 } & \multicolumn{15}{|c|}{ Moyennes } \\
\hline & 0,19 & 0,29 & I & 0,07 & 0,00 & O,I 9 & 0,23 & 0,05 & 0,36 & 0,36 & $0,7 \mathrm{I}$ & 0,15 & 0,44 & $0,1 \mathrm{I}$ & 0,64 \\
\hline & 0,18 & 0,28 & I & 0,08 & 0,00 & 0,17 & 0,27 & 0,07 & 0,33 & 0,35 & 0,72 & 0,13 & 0,39 & $0,1 \mathrm{I}$ & 0,67 \\
\hline & 0,17 & 0,27 & I & 0,09 & $0,0 I$ & 0,17 & 0,20 & 0,08 & 0,30 & $0,3^{8}$ & 0,73 & 0,17 & 0,42 & 0,15 & 0,70 \\
\hline & 0,14 & $0,3 \mathrm{I}$ & I & 0,10 & 0, or & $0, I_{7}$ & 0,04 & 0,07 & 0,33 & 0,26 & 0,69 & 0,15 & 0,34 & $0, \mathbf{I}$ I & 0,67 \\
\hline & 0,15 & $0,3 \mathrm{I}$ & I & 0,09 & $0, \mathrm{OI}$ & 0,17 & 0,06 & 0,07 & 0,33 & 0,28 & 0,69 & 0,14 & 0,35 & 0,10 & 0,67 \\
\hline \multirow{6}{*}{$\begin{array}{ll}\text { I Machines } . . . & . \\
2 & \text { Fonderies . . . } \\
3 & \text { Bâtiment . . } \\
4 & \text { Tunnels et galeries } \\
5 & \text { Carrières . . . . }\end{array}$} & \multicolumn{15}{|c|}{ Variances } \\
\hline & 0,53 & 0,54 & 1,12 & 0,10 & 0,00 & 0,42 & 0,26 & 0,07 & 0,79 & 1,03 & $I, 03$ & 0,16 & 0,78 & 0,13 & 1,59 \\
\hline & 0,54 & 0,42 & 0,95 & 0, ro & 0,00 & 0,43 & 0,26 & 0,09 & 0,58 & 0,88 & 0,76 & O, I 5 & 0,66 & 0,13 & 1,17 \\
\hline & 0,58 & 0,50 & 0,97 & $0, \mathrm{II}$ & $0,0 I$ & $0,5^{\circ}$ & 0,22 & 0, Io & 0,63 & $\mathrm{I}, 04$ & 0,93 & 0,17 & 0,86 & 0,16 & $\mathrm{r}, 25$ \\
\hline & 0,50 & 0,86 & $I, 27$ & 0,10 & $0,0 \mathrm{I}$ & $0,5^{2}$ & 0,07 & 0,08 & $\mathrm{I}, 04$ & 0,73 & $I, 23$ & 0,14 & 0,75 & 0,12 & $\mathrm{I}, 53$ \\
\hline & 0,59 & 1,09 & $\mathrm{I}, 04$ & 0, I I & $0,0 \mathrm{I}$ & 0,59 & 0,08 & 0,09 & 1,30 & 0,79 & $\mathrm{I}, 49$ & 0,13 & 0,78 & $0,1 \mathrm{I}$ & $\mathrm{r}, 82$ \\
\hline \multirow{6}{*}{ 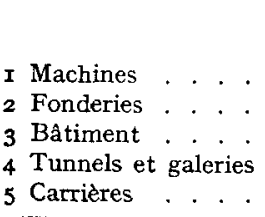 } & \multicolumn{15}{|c|}{ Asymétries } \\
\hline & 22,9 & $27, I$ & 16,2 & 3,9 & $\mathrm{I}, 4$ & I 6,6 & 15,7 & 3,9 & 35,4 & $14, I$ & 14,3 & 3,5 & I I , I & 3,4 & I 6,4 \\
\hline & 17,2 & 7,8 & 8,4 & 3,0 & 0,8 & I $3, I$ & 8,3 & $3, \mathrm{I}$ & 9,6 & I I, 9 & 4,3 & 2,7 & $10, I$ & 2,7 & 4,6 \\
\hline & I 6,5 & I6, I & $8, \mathrm{I}$ & $3, \mathrm{I}$ & 2,5 & I 3,4 & II 1,4 & $3, \mathrm{I}$ & r 9,0 & 13,2 & 8,3 & 3,0 & I 1,4 & 3,0 & 8,5 \\
\hline & 14,4 & 30,3 & I I , 9 & 2,4 & 2,8 & $\mathrm{I} 2, \mathrm{I}$ & 12,9 & 2,5 & 35,5 & $\mathrm{I} 2,7$ & 29,4 & 2,0 & I I , I & 2,0 & $3 I, 5$ \\
\hline & 14,0 & $x 8,8$ & 8,3 & 3,0 & $\mathbf{I}, 8$ & II,7 & 9,9 & 3,2 & 22,6 & I 5,6 & 15,4 & 2,6 & 10,0 & 2,6 & $\mathbf{1 7 , 5}$ \\
\hline
\end{tabular}




$$
\begin{aligned}
& \text { Variance }=\sqrt{M_{2}}=\sqrt{\sum_{z=0}^{\infty}\left(z-m_{1}\right)^{2} u(z)} \\
& \text { Asymétrie }=\frac{M_{3}}{M_{2}^{3}{ }^{3 / 2}}=\frac{\sum_{z-0}^{\infty}\left(z_{1}-m_{1}\right)^{3} u(z)}{M_{2}^{3 / 2}}
\end{aligned}
$$

Le coût de chaque catégorie est normalisé à la moyenne du coût

\begin{tabular}{|c|c|c|c|c|c|c|c|c|c|}
\hline \multirow{2}{*}{ Groupes } & \multicolumn{9}{|c|}{ Coût total moyen d'un accident en fr.s. } \\
\hline & $T$ & $\mathrm{TCr}$ & $T c r$ & $T C R$ & $T c R$ & $T C$ & $T c$ & $T R$ & $\operatorname{Tr}$ \\
\hline I Machines & 283 & 276 & $2 \mathrm{I}$ & 7888 & 45489 & 518 & 94 & IO I00 & I33 \\
\hline 2 Fonderies & 398 & $3 \mathrm{I} 2$ & 23 & I I 667 & $4440 I$ & 670 & 85 & I 2888 & I75 \\
\hline 3 Bâtiment & 484 & 323 & 23 & 13712 & 27058 & 705 & I 16 & 14612 & 209 \\
\hline 4 Tunnels et galeries & I 135 & 467 & 24 & 19165 & 42689 & I 303 & 779 & 22877 & 322 \\
\hline 5 Carrières. . . & 772 & 371 & 23 & 13082 & 26678 & 923 & 455 & I 5 I 27 & 257 \\
\hline
\end{tabular}
total d'un accident de cette catégorie calculée sur la base de la

4

\begin{tabular}{|c|c|c|c|c|c|c|c|c|c|c|c|c|c|c|c|}
\hline \multicolumn{6}{|c|}{ Coût des rentes } & \multicolumn{9}{|c|}{ Cont total } & \multirow{2}{*}{ Groupes } \\
\hline$T$ & $T C R$ & $T c R$ & $T C$ & $T c$ & $T R$ & $T$ & $\mathrm{TCr}$ & $T_{c r}$ & $T C R$ & $T c R$ & $T C$ & $T c$ & $T R$ & $T r$ & \\
\hline \multicolumn{15}{|c|}{ Moyennes } & \multirow{6}{*}{ 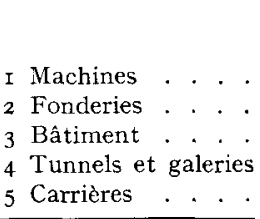 } \\
\hline 0,45 & 0,78 & $I, 00$ & 0,37 & 0,77 & 0,84 & I & I & I & $\mathbf{I}$ & I & I & I & I & $I$ & \\
\hline 0,47 & 0,79 & 1,00 & 0,44 & 0,73 & 0,82 & I & I & I & $\mathbf{I}$ & I & I & $\mathbf{I}$ & I & I & \\
\hline 0,45 & 0,74 & 0,99 & $0,4 \mathrm{I}$ & 0,80 & 0,77 & I & I & $\mathbf{I}$ & I & I & I & $\mathbf{I}$ & I & I & \\
\hline 0,60 & 0,75 & 0,99 & 0,49 & 0,96 & 0,82 & I & I & $\mathbf{I}$ & $\mathbf{I}$ & I & I & I & I & I & \\
\hline 0,57 & 0,77 & 0,99 & 0,48 & 0,94 & 0,83 & I & I & I & $\mathbf{I}$ & I & I & I & $\mathbf{I}$ & I & \\
\hline \multicolumn{15}{|c|}{ Variances } & \multirow{6}{*}{ 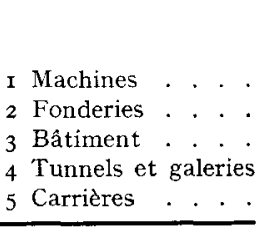 } \\
\hline 6,95 & $\mathrm{I}, \mathrm{O} 3$ & 0,58 & 3,48 & 22,30 & 1,36 & 7,53 & $\mathrm{I}, 49$ & $\mathrm{I}, \mathrm{I} 2$ & $\mathrm{I}, \mathrm{I} 7$ & 0,57 & 4,16 & 22,32 & I,39 & 2,26 & \\
\hline $5,9^{\circ}$ & $I, 07$ & 0,36 & $4, \mathrm{II}$ & 20,72 & $\mathrm{I}, \mathrm{II}$ & 6,66 & $\mathrm{I}, \mathrm{IO}$ & 0,95 & $I, 20$ & 0,36 & 4,78 & 20,74 & $\mathrm{I}, 20$ & 1,65 & \\
\hline 4,65 & 0,73 & 0,77 & 3,39 & 17,24 & $0,8 \mathrm{I}$ & 5,65 & $\mathrm{I}, 34$ & 0,97 & 0,89 & 0,77 & 4,36 & $\mathrm{I} 7,3 \mathrm{O}$ & 0,92 & 1,78 & \\
\hline 4,80 & $0,9 \mathrm{I}$ & 0,66 & 3,62 & 8,64 & 0,96 & 5,42 & 2,00 & 1,27 & $\mathrm{I}, 05$ & $o, 66$ & 4,47 & 8,67 & $\mathrm{I}, \mathrm{O} 2$ & 2,47 & \\
\hline 4,54 & 0,89 & $0,7 \mathrm{I}$ & 3,44 & 9,05 & 0,94 & 5,12 & 2,48 & $\mathrm{I}, \mathrm{O} 4$ & $I, O O$ & $0,7 \mathbf{I}$ & 4,18 & 9,08 & 0,98 & 3,00 & \\
\hline \multicolumn{15}{|c|}{ Asymétries } & \multirow{6}{*}{$\begin{array}{ll}\text { I } & \text { Machines . . . } \\
2 & \text { Fonderies . . . } \\
3 & \text { Bâtiment . . . } \\
4 & \text { Tunnels et galeries } \\
5 & \text { Carrières . . . . }\end{array}$} \\
\hline 26,1 & 2,3 & $-0, \mathbf{I}$ & 14,1 & 32,0 & $3, \mathrm{I}$ & 22,9 & 20,1 & I 6,2 & 2,3 & $-\mathrm{O}, \mathrm{I}$ & I 3,2 & 32,0 & 2,8 & 23,8 & \\
\hline I 9,7 & 2,8 & o & 15,8 & 30,8 & 2,4 & I7, 8 & 5,4 & 8,4 & 2,5 & $-0, \mathrm{I}$ & $\mathrm{I} 4,2$ & 30,8 & 2,2 & 5,8 & \\
\hline I 5,9 & 2,0 & 0,5 & I 2,4 & 25,6 & $2, \mathrm{I}$ & I 3,7 & 9,7 & $8, \mathrm{I}$ & $\mathrm{I}, 8$ & 0,5 & I I, 2 & 25,5 & $\mathrm{I}, 7$ & I 0,4 & \\
\hline I I, 5 & 2,3 & 0,5 & I I, 6 & 10,6 & I, 8 & I0, 4 & 29,9 & I I, 9 & $2, \mathrm{I}$ & 0,5 & 10,2 & 10,6 & $\mathrm{I}, 7$ & 33,3 & \\
\hline 12,2 & 2,3 & $\mathrm{I}, \mathrm{I}$ & 11,4 & 12,3 & $2, I$ & Io, 8 & I $6, \mathrm{I}$ & 8,3 & 2,2 & $\mathrm{I}, \mathrm{I}$ & 9,9 & 12,3 & 2,0 & 18,7 & \\
\hline
\end{tabular}

des fréquences $u(z)$ 
table I. Nous indiquons ci-après les moyennes de normalisation utilisées.

Fonctions de distribution $\mathrm{U}(\mathrm{z})$ et $\mathrm{S}(\mathrm{z})$

(Groupes I-5, ajustement graphique, en pour-cent)

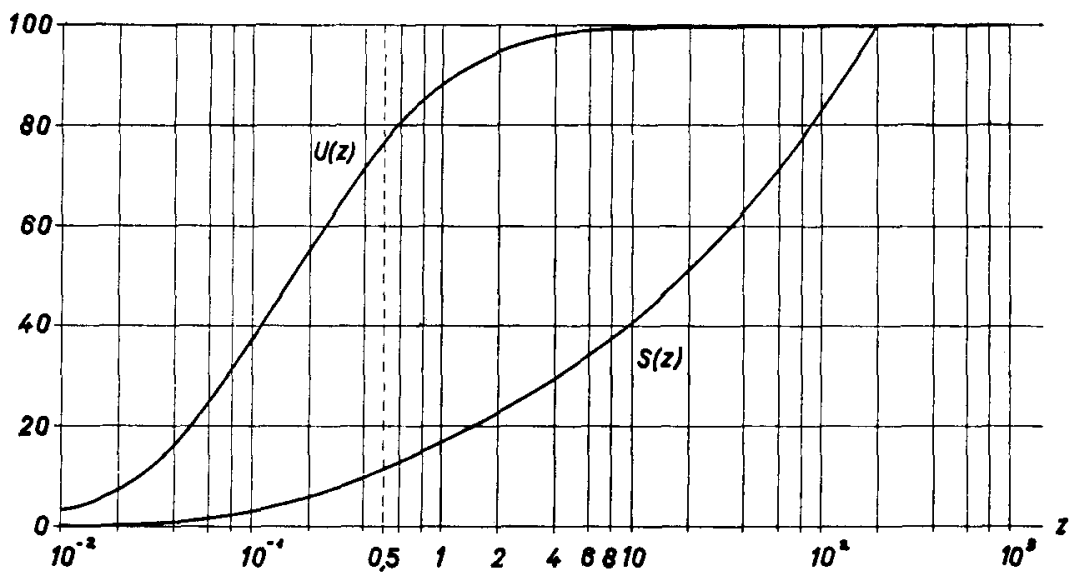

Comme il ressort de la table 4, les moyennes des frais normalisés des différents groupes concordent au sein de toutes les catégories, à l'exception de celles des groupes 4 et 5 de la catégorie Tc pour le coût des frais de traitement et des rentes. Pour les variances, les valeurs des groupes I- 3 d'une part et 4-5 d'autre part se rapprochent beaucoup. Il est intéressant de relever que l'image des asymétries est très variée. Les différences sont particulièrement importantes entre les groupes pour les catégories n'ayant pas de charge due aux rentes. Un autre fait remarquable est la présence de petites asymétries négatives.

5. L'examen des distributions du coût d'un accident soulève de nombreux problèmes. Parmi ceux, généralement encore non résolus, qui sont d'un intérêt particulier pour la solution des questions qui se posent dans l'application de la théorie du risque, nous pouvons mentionner les suivants: 
Dépendance de la distribution du coût d'un accident des variations dans la grandeur et la structure de l'effectif assuré

variations dans la fréquence et le montant des prestations ultérieures modifications du salaire maximum assuré

déplacements de poids qui se produisent dans les parts des composantes de la charge ou des catégories d'accidents.

Variations des fonctions de distribution dues à la survenance ou à l'absence d'accidents graves et coûteux.

Dépendance entre les composantes de la charge. Mise en évidence des facteurs déterminant la convolution des composantes représentée par la distribution empirique du coût total d'un accident.

Caractère représentatif des fonctions de répartition au point de vue de la théorie des sondages.

6. Le but des examens des distributions empiriques du coût d'un accident dont nous venons de parler n'est pas la représentation analytique de ces distributions sous une forme explicite. Une telle représentation est d'ailleurs extrêmement difficile, d'une part à cause des nombreux accidents qui n'occasionnent que des coûts minimes, d'autre part à cause des cas de rentes, peu nombreux, mais qui au point de vue coût pèsent d'autant plus dans la balance. Notre but est au contraire de montrer les différences fondamentales qui existent entre les différentes répartitions ou leurs propriétés communes. Le résultat de ces examens a une certaine importance pour la solution du problème assez difficile que représente la détermination des primes dans l'assurance-accidents obligatoire en Suisse. Nous renvoyons tout particulièrement à ce sujet au chapitre „L'accident, un processus aléatoire" du rapport mentionné dans notre introduction sur les ,Résultats de la statistique des accidents de la huitième période quinquennale 1953-1957". 